\title{
Adaptive Resource Allocation for Multicast OFDM Systems with Multiple Transmit Antennas
}

\author{
Berna Özbek ${ }^{{ }^{*}}$, Didier Le Ruyet ${ }^{\dagger}$ and Hajer Khiari ${ }^{\dagger}$ \\ $\dagger$ Electronics and Communications Laboratory, CNAM, 292 rue Saint Martin, 75141, Paris, France \\ e-mail: \{ozbek,leruyet,khiari\}@cnam.fr \\ * Electrical and Electronics Engineering Department, Izmir Institute of Technology, Urla, 35430, Izmir, Turkey \\ e-mail: bernaozbek@iyte.edu.tr
}

\begin{abstract}
We evaluate the impact of multiple transmit antennas on the performance of multicast OFDM systems by proposing a suboptimal low complexity algorithm. Associated with a powerful erasure code, it is possible to increase the data rate of multicast OFDM systems by selecting for each subcarrier to the users with a good channel condition. We show that the resource allocation which includes the precoding vector selection, subcarrier allocation and bit loading is a difficult optimization problem. We propose a suboptimal algorithm to solve this problem by avoiding the optimization to reduce complexity. When the users are not symmetrically distributed around the base station, we add a fair scheduler to guarantee that each user receives the same amount of data. We present simulation results where we compare the proposed multicast systems with the classical multicast OFDM systems. When the users are symmetrically distributed around the base station, the gain is rather small, whereas when the users are non-symmetrically distributed, the proposed algorithm outperforms OFDM systems with subcarrier allocation.
\end{abstract}

keywords : Multicast OFDM, multiple transmit antennas, precoding vector optimization, adaptive subcarrier allocation.

\section{INTRODUCTION}

Up to now, the main wireless applications are broadcast multiuser systems. However, the demand for the multimedia services such as video and audio conferencing, online training, news and software distribution and database replication is increasing. For these applications, multicasting offers a significant improvement compared to broadcasting since it allows the transmission of packets to multiple destinations using less resources [1]. However, since in the wireless channel the received signal-to-noise ratio (SNR) of each user is not the same, the data rate of the multicast stream is limited by the data rate of the least capable user. Consequently, this method cannot provide efficient performance when the number of users in the group increases.

The main difficulty in achieving high data rate on the wireless channels is known to be frequency selectivity and the fading due to the existence of multiple paths. Orthogonal frequency division multiplexing (OFDM) techniques can significantly alleviate the impacts of frequency selective fading [2] and is attractive for the next generation of wireless systems. Moreover, multiple-input single output (MISO) systems that use multiple antenna techniques [3] are combined with OFDM systems to enhance the performance of wireless systems in fading channels.

In [4] [5], by assuming that the transmitter knows the instantaneous channel transfer function of all the users, it has been shown that adaptive subcarrier and bit allocation can significantly increase the data rate of broadcast multiuser OFDM scheme. Recently it has been demonstrated in [6] that using OFDM with subcarrier and bit allocation, it is also possible to increase the data rate on each subcarrier for multicasting applications by selecting only the users with a good channel condition.

In this paper, we propose to evaluate the impact of multiple transmit antennas on the performance of multicast OFDM systems. Our purpose is to maximize the sum data rate to increase the quantity of received data by all users for a given time instead of maximizing the worst user rate. We show the determination of the precoding vector, allocation of the subcarriers to the users and loading bits to the subcarriers is an optimization problem. We propose a suboptimal algorithm to solve this complex problem. With these algorithms, we allow one subcarrier to carry data for more than one user by optimizing the precoding vector. This approach can be combined with a fair scheduler and a powerful erasure codes such as digital fountain codes [7].

The basic principle behind the use of erasure codes is that the original source data is encoded in the form of a sequence of packets. These packets are transmitted to the users. A receiver can reconstruct the original source data once it receives a sufficient number of encoded packets. The main benefit of this approach is that different receivers can recover the source data using different encoded packets. Therefore, our purpose is to maximize the total number of received packets using precoding vector optimization, subcarrier allocation and bit loading while providing the reception of almost the same amount of packets to each user using a fair scheduler.

This paper is organized as follows. First, we describe the system model of multicast OFDM systems with multiple transmit antennas over wireless channels in section II. Then, we propose the precoding vector optimization, subcarrier allocation and bit loading algorithms including fair scheduling in section III. Finally, we give simulation and comparison results in section IV. 


\section{System MOdEl FOR Multicast OfDM With MULTIPLE TRANSMIT ANTENNAS}

The multicast OFDM system where the base station with $N_{t}$ transmit antennas serves $K$ users with a single receive antenna is considered as shown in Figure 1. The common data is formed into the OFDM symbol with $N$ subcarriers and then transmitted from $N_{t}$ antennas through the frequency selective channels. For each $p$ th frame, the channel that is between the $j$ th transmit antenna and $k$ th user is described using a baseband equivalent impulse response as

$$
\mathbf{h}_{p, k, j}=\left[\begin{array}{llll}
h_{p, k, 1, j} & h_{p, k, 2, j} & \ldots & h_{p, k, L_{f}, j}
\end{array}\right]^{T}
$$

where $L_{f}$ is the length of the channel response. The channel is assumed to be constant over one frame and varying between the frames considering Doppler frequency. Moreover, it is assumed that the channel taps is equal or smaller than the length of the guard interval (GI) in order to avoid intercarrier and intersymbol interference at the receiver.

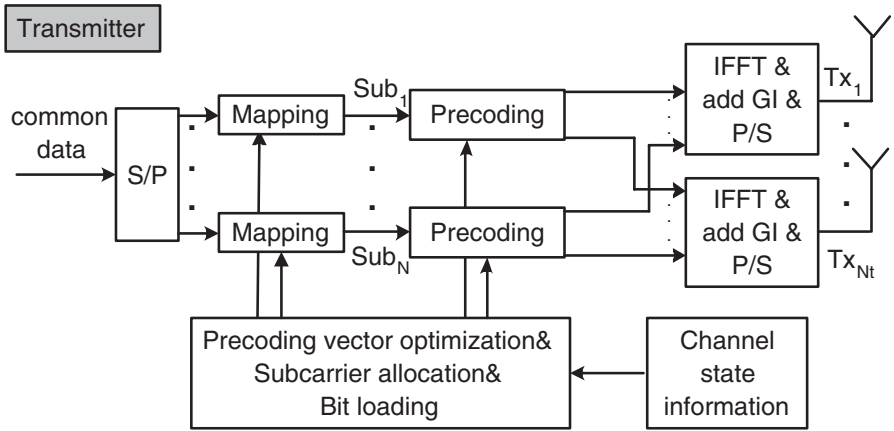

\section{$k^{\text {th }}$ user receiver}

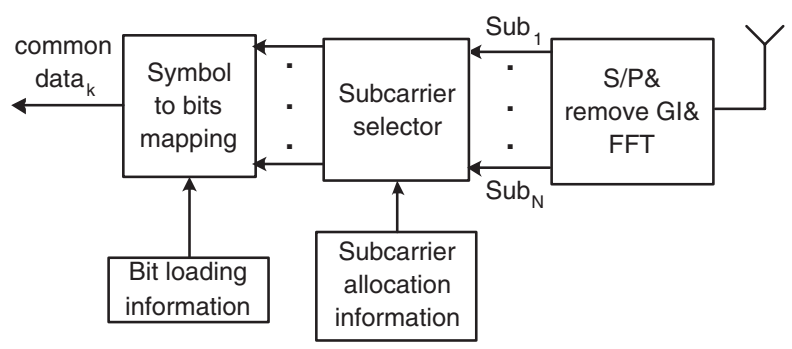

Fig. 1. The transmitter and $k$ th receiver structure for multicast OFDM system with multiple transmit antennas.

Using equation (1), the channel vector for $k$ th user and $n$th subcarrier in the frequency domain is written by

$$
\mathbf{H}_{p, k, n}=\left[\begin{array}{llll}
H_{p, k, n, 1} & H_{p, k, n, 2} & \ldots & H_{p, k, n, N_{t}}
\end{array}\right]^{T}
$$

where $H_{p, k, n, j}$ is the channel gain from $j$ th transmit antenna to $k$ th user for $n$th subcarrier.

Assuming that the channel information about all the subcarriers for all users are known at the transmitter, the adaptive resource allocation should optimize the precoding vector, allocate the users to the subcarrier and load bits in a way that maximizes the total number of bits received by all the users. Each subcarrier is assigned to a group of users which receive the same data, and then the number of bits on each subcarrier is determined considering the lowest one among the channel gains of all the users allocated to this subcarrier. It is assumed that the subcarrier/bit allocation information is transmitted to each user through a separate control channel.

Before defining the optimization problem, first the notations will be given. Let $R_{p, k}$ be the data rate of the $k$ th user and $c_{p, n}$ be the number of bits that are assigned to the $n$th subcarrier. Here, the user index is unnecessary because the users using the subcarrier receive identical data using the same modulation. $c_{p, n}$ is selected from the set of $\{0,1,2, \ldots, M\}$ where $M$ is the maximum number of bits per symbol that can be transmitted by each subcarrier $n=1,2, \ldots, N$.

The data rate $R_{p, k}$ can be expressed as

$$
R_{p, k}=\sum_{n=1}^{N} c_{p, n} \rho_{p, k, n}
$$

where $\rho_{p, k, n}$ is a binary value indicating whether $k$ th user utilizes the $n$th subcarrier or not.

$$
\rho_{p, k, n}= \begin{cases}1 & \text { if the nth subcarrier is used for kth user } \\ 0 & \text { else }\end{cases}
$$

Assuming that available total transmit power (in energy per symbol) is limited by $P_{T}$, in order to maximize the total data rate by all users, the optimization problem can be expressed as

$$
\max _{\mathbf{W}_{p} ; c_{p} ; \rho_{p}} \sum_{k=1}^{K} R_{p, k}=\max _{\mathbf{W}_{p} ; c_{p} ; \rho_{p}} \sum_{k=1}^{K} \sum_{n=1}^{N} c_{p, n} \rho_{p, k, n}
$$

subject to

$$
\sum_{n=1}^{N} \max _{k}\left(\frac{f\left(c_{p, n}\right) \rho_{p, k, n}}{\left\|\mathbf{W}_{p} \mathbf{H}_{p, k, n}\right\|^{2}}\right) \leq P_{T}
$$

where $\mathbf{W}_{p}$ is the $1 \times N_{t}$ precoding vector as shown in Figure 2 and $f\left(c_{p, n}\right)$ is the required received power (in energy per symbol) for reliable reception of $c_{p, n}$ bits when the channel gain is equal to unity.

For M-ary quadrature amplitude modulation (M-QAM) schemes, the bit error probability is upper bounded by the symbol error probability, which is tightly approximated by $4 Q\left(\sqrt{d^{2} /\left(2 N_{0}\right)}\right)$ [8] where $d$ is the minimum distance between the points in the signal constellation. Since the average energy of a M-QAM symbol is equal to $(M-1) d^{2} / 6$, then the required power (in energy per symbol) $f\left(c_{p, n}\right)$ for supporting $c_{p, n}$ bits per symbol at a required bit-error-rate (BER) $p_{e}$ can be represented by

$$
f\left(c_{p, n}\right)=\frac{N_{0}}{3}\left[Q^{-1}\left(p_{e} / 4\right)\right]^{2}\left(2^{c_{p, n}}-1\right)
$$

where $N_{0} / 2$ denotes the variance of the additive white Gaussian noise (AWGN) and $Q(x)=\frac{1}{\sqrt{2 \pi}} \int_{x}^{\infty} \mathrm{e}^{-t^{2} / 2} d t$. 


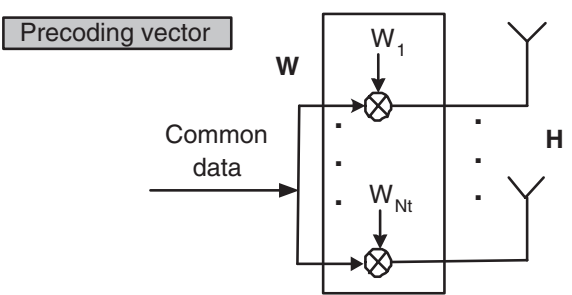

Fig. 2. The block diagram of the precoding vector for OFDM with multiple transmit antennas.

\section{Adaptive Resource Allocation Algorithms}

The problem includes the precoding vector optimization, subcarrier allocation and bit loading. Since, it is very difficult to solve (5) subject to (6), we propose to separate bit allocation problem and solve both the precoding vector optimization and subcarrier allocation by assuming equal power for each subcarrier. Therefore, the user data rate should be maximized for each subcarrier. Then, the expression of the new suboptimal optimization can be written as

For each subcarrier $n$ :

$$
\max _{\mathbf{W}_{p, n} ; c_{p, n} ; \rho_{p, n}} \sum_{k=1}^{K} c_{p, n} \rho_{p, k, n}
$$

subject to

$$
\max _{k}\left(\frac{f\left(c_{p, n}\right) \rho_{p, k, n}}{\left\|\mathbf{W}_{p, n} \mathbf{H}_{p, k, n}\right\|^{2}}\right) \leq \frac{P_{T}}{N}
$$

This optimization problem can be solved for each subcarrier $n$ by considering all the $2^{K}-1$ possible user allocations and by searching the best precoding vector associated to each case. Let $\boldsymbol{\rho}_{p, 1, n}, \boldsymbol{\rho}_{p, 2, n}, \ldots, \boldsymbol{\rho}_{p, i, n}, \ldots, \boldsymbol{\rho}_{p, 2^{K}-1, n}$ be the set of the non-zero vectors of the $K$ dimensional hypercube as follows:

$$
\begin{aligned}
& \boldsymbol{\rho}_{p, 1, n}=(1,0, \ldots, 0), \boldsymbol{\rho}_{p, 2, n}=(0,1, \ldots, 0), \ldots, \\
& \boldsymbol{\rho}_{p, K, n}=(0,0, \ldots, 1), \ldots, \boldsymbol{\rho}_{p, 2^{K}-1, n}=(1,1, \ldots, 1)
\end{aligned}
$$

For each subcarrier $n$ :

For each index $i$ :

Step 1) Optimization of the precoding vector (max-min algorithm):

$\max _{\mathbf{W}_{p, i, n}} \min \left\{\mathbf{H}_{p, k^{\prime}, n}^{H} \mathbf{W}_{p, i, n}^{H} \mathbf{W}_{p, i, n} \mathbf{H}_{p, k^{\prime}, n} ; k^{\prime} \in \mathcal{K}\right\}$

subject to

$$
\mathbf{W}_{p, i, n} \mathbf{W}_{p, i, n}^{H} \leqslant 1
$$

where $\mathcal{K}=\left\{k \mid \rho_{p, k, i, n}=1\right\}$

Step 2) Calculation of the gains:

$$
\mathbf{G}_{p, k^{\prime}, i, n}=\left\|\mathbf{W}_{p, i, n} \mathbf{H}_{p, k^{\prime}, n}\right\|^{2}
$$

where $k^{\prime} \in \mathcal{K}$.

Step 3) Determination of the number of bits allocated and the data rate:
Let $c_{p, i, n}$ be the number of bits that can be received by the selected users at the $n$th subcarrier. The number of supportable bits is given by

$$
c_{p, i, n}=\min \left(f^{-1}\left(\frac{P_{T}}{N} \min _{k^{\prime}}\left(\mathrm{G}_{p, k^{\prime}, i, n}\right)\right), M\right)
$$

where $f^{-1}($.$) is the inverse function of f($.$) defined$ in (7). Calculate the total data rate $\bar{R}_{p, i, n}$.

$$
\bar{R}_{p, i, n}=\left(\sum_{k=1}^{K} \rho_{p, k, i, n}\right) c_{p, i, n}
$$

End)

Step 4) Selection of the index that maximizes the sum data rate:

Select the index $\kappa$ maximizing $\bar{R}_{p, i, n}$.

$$
\kappa=\arg \max _{i} \bar{R}_{p, i, n}
$$

Then, the precoding vector is chosen as

$$
\mathbf{W}_{p, n}=\mathbf{W}_{p, \kappa, n}
$$

Step 4) Allocation of the users to the subcarrier:

$$
\rho_{p, k, n}=\rho_{p, k, \kappa, n}
$$

Since the max-min algorithm is NP-hard, the problem can be only solved approximately. Following [9], using relaxation, the problem is transformed into a suitable form for semidefinite programming. Then, using interior point method, the optimization is performed. Except for small value of $K$, this optimization problem is intractable. As a consequence, we propose a suboptimal algorithm to optimize the precoding vector and to choose the configuration of user allocation.

\section{A. Proposed Algorithm}

In this section, we propose to choose the precoding vector among a limited set of precoding vectors. This set is composed of the normalized transpose-conjugate of the channel vectors associated to each user. The selected precoding vector and user allocation will maximize the sum data rate.

For each subcarrier $n$ :

Step 1) Initializing of the precoding vector:

$$
\mathbf{W}_{p, i, n}^{\text {init }}=\frac{\left[\begin{array}{llll}
H_{p, i, n, 1}^{*} & H_{p, i, n, 2}^{*} & \ldots & H_{p, i, n, N_{t}}^{*}
\end{array}\right]}{\sqrt{\left|H_{p, i, n, 1}\right|^{2}+\ldots\left|H_{p, i, n, N_{t}}\right|^{2}}}
$$

where $i=1,2, \ldots, K$.

\section{Step 2) Calculation of the gains:}

Then, the gain of each user for each initial precoding vector is calculated as

$$
\mathrm{G}_{p, k, i, n}=\left\|\mathbf{W}_{p, i, n}^{\mathrm{init}} \mathbf{H}_{p, k, n}\right\|^{2}
$$

Let $c_{p, k, i, n}$ be number of bits that can be received by $k$ th user using $i$ th initial precoding vector in the 
case of $\rho_{p, k, n}=1$. The number of supportable bits is given by

$$
c_{p, k, i, n}=\min \left(f^{-1}\left(\frac{P_{T}}{N} \mathrm{G}_{p, k, i, n}\right), M\right)
$$

Step 3) Selection of both the precoding vector and the user that maximize the sum data rate:

Calculate the tentative total data rate $\bar{R}_{p, k, i, n}$ when the $k$ th user is selected as the user requiring the maximum power for each initial precoding vector.

$$
\bar{R}_{p, k, i, n}=u_{p, k, i, n} c_{p, k, i, n}
$$

where $u_{p, k, i, n}$ indicates the number of users who have channel gains larger than $\mathrm{G}_{p, k, i, n}$. Select the user index $\kappa$ and the precoding vector index $\varrho$ maximizing $\bar{R}_{p, k, i, n}$.

$$
\kappa, \varrho=\arg \max _{k, i} \bar{R}_{p, k, i, n}
$$

Then, the precoding vector is chosen as

$$
\mathbf{W}_{p, n}=\mathbf{W}_{p, \varrho, n}^{\mathrm{init}}
$$

Step 4) Allocation of the users to the subcarrier:

$$
\rho_{p, k, n}= \begin{cases}1 & \text { if } \quad \mathrm{G}_{p, k, \varrho, n} \geq \mathrm{G}_{p, \kappa, \varrho, n} \\ 0 & \text { else }\end{cases}
$$

\section{B. Proposed Fair Scheduling Algorithm}

Our purpose is that each user receives almost the same amount of packets at the end of transmission in the case of non-symmetric user distribution around the base station. Therefore, we should use a fair scheduling algorithm. For each user, we define a priority factor that represents the ratio between the received bits by that user and the total transmitted bits. The priority factor is inversely proportional to the total number of bits received by the user.

$$
P_{p, k}=\frac{\sum_{p^{\prime}=1}^{p-1} \sum_{k_{x}=1}^{K} R_{p^{\prime}-1, k_{x}}}{\sum_{p^{\prime}=1}^{p-1} R_{p^{\prime}-1, k}}
$$

where $p-1$ is the index of total transmitted frame. The priority vector is reconstructed from the priority factors of all users after normalization.

$$
\mathbf{P}_{p}=\frac{1}{\sum_{k=1}^{K} P_{p, k}^{\alpha}}\left[\begin{array}{llll}
P_{p, 1}^{\alpha} & P_{p, 2}^{\alpha} & \ldots & P_{p, K}^{\alpha}
\end{array}\right]
$$

where $\alpha$ is the parameter that accelerate the convergence time of the algorithm for the delay sensitive applications.

For the proposed algorithm, the step 3 is changed to include the priority factor as
Step 3) Selection of the precoding vector and user that maximize the sum data rate:

Calculate the rational total data rate $\bar{O}_{p, k, i, n}$ when the $k$ th user is selected as the user requiring maximum power for each initial precoding vector.

$$
\bar{O}_{p, k, i, n}=\mathbf{P}_{p} \mathbf{U}_{p, i, n} c_{p, k, i, n}
$$

where $\mathbf{U}_{p, i, n}=\left[\begin{array}{lll}U_{p, 1, i, n} & \ldots U_{p, k_{y}, i, n} \ldots & U_{p, K, i, n}\end{array}\right]^{T}$ and

$$
U_{p, k_{y}, i, n}= \begin{cases}1 & \text { if } \quad \mathrm{G}_{p, k_{y}, i, n} \geq \mathrm{G}_{p, k, i, n} \\ 0 & \text { else }\end{cases}
$$

Then, the selection of the user index is performed by maximizing $\bar{O}_{p, k, i, n}$.

$$
\kappa^{f}, \varrho^{f}=\arg \max _{k, i} \bar{O}_{p, k, i, n}
$$

In order to evaluate the fairness of each algorithm, we consider the fairness index (FI) given by [10]

$$
\mathrm{FI}=\frac{\left(\sum_{k=1}^{K} R_{k}\right)^{2}}{K \sum_{k=1}^{K} R_{k}^{2}}
$$

where $R_{k}=\sum_{p=1}^{P} R_{p, k}$ and $P$ is the number of total frame at the end of transmission. The FI ranges between 0 (no fairness) and 1 (perfect fairness).

\section{Bit Loading Algorithm}

Bit loading algorithm is considered using the modified Levin-Campello algorithm [4] [6] under the assumption that the subcarrier allocation is completed. Let $\Delta P_{p, n}(c)$ denote the incremental power needed for the transmission of one additional bit at the subcarrier $n$. When the number of loaded bits for the $n$th subcarrier is $c, \Delta P_{p, n}(c)$ is given for the proposed algorithm

$$
\Delta P_{p, n}(c)=\frac{P_{T}}{N} \frac{f(c+1)-f(c)}{u_{p, n} \mathrm{G}_{p, \kappa, \rho, n}}
$$

where $u_{p, n}$ is the number of users who share the $n$th subcarrier, which is necessary because the incremental power is shared by the group of users allocated to the subcarrier.

$$
u_{p, n}=\sum_{k=1}^{K} \rho_{p, k, n}
$$

Then, the bit loading algorithm is summarized as follows:

Step 1) Initialization:

$c_{p, n}=0$ and evaluate $\Delta P_{p, n}(c=0)$ for all $n$.

Tentative transmit power is $P_{T}^{*}=0$.

Step 2) Bit Loading Iteration:

repeat the following unless $P_{T}^{*} \geq P_{T}$

$n^{*}=\min \Delta P_{p, n}\left(c_{p, n}\right)$

$P_{T}^{*}=\stackrel{n}{P_{T}^{*}}+\Delta P_{p, n^{*}}\left(c_{p, n^{*}}\right) u_{p, n^{*}}$ 
$c_{p, n^{*}}=c_{p, n^{*}}+1$

if $c_{p, n^{*}}=M$, set $\Delta P_{p, n^{*}}\left(c_{p, n^{*}}\right)=\infty$

else evaluate $\Delta P_{p, n^{*}}\left(c_{p, n^{*}}\right)$.

\section{NumericAl Results}

In this section, we evaluate the sum data rate of the multicast OFDM systems with multiple transmit antennas using the proposed algorithm and compare it with the one of the maxmin worst user algorithm. This algorithm is performed using Step 1 that includes max-min optimization algorithm only considering the vector $\boldsymbol{\rho}_{p, 2^{K}-1, n}$ and bit loading. Since each subcarrier is allocated to all the users, a fair scheduler is not necessary. We also give the comparison results with the multicast OFDM systems by using worst user and subcarrier allocation algorithms [6].

Simulation results are performed using Hiperlan/2 standard [11] using channel model A [12] which corresponds to a typical office environment with 9 channel taps. The total OFDM symbol duration is $4 \mu \mathrm{s}$ including $0.8 \mu \mathrm{s}$ guard interval. It consists of $N=64$ subcarriers. The required BER, the noise variance and the maximum number of bits per symbol are chosen as $p_{e}=10^{-4}, N_{0}=1$ and $M=8$ respectively. $P$ is chosen as 1000 and the duration of one frame is selected as $800 \mathrm{~ms}$. The transmitted power is fixed at $P_{T}=27 \mathrm{dBW}$.

In Figure 3, we show the performance results using two transmit antennas and assuming that the users are symmetrically distributed around the base station without path loss. The results indicate that the sum data rate is increased by $15 \%$ using the proposed algorithm compared to OFDM systems with subcarrier allocation [6]. Furthermore, the max-min worst user algorithm outperforms the OFDM worst user algorithm.

In Figure 4, we draw the simulation results using two transmit antennas and assuming that the users are nonsymmetrically distributed around the base station and the path loss of users is chosen from the set $[0 \mathrm{~dB}, 5 \mathrm{~dB}, 10 \mathrm{~dB}, 15 \mathrm{~dB}]$ with the ratio of $25 \%$. In this scheme, a fair scheduler should be added to guaranty that each user receives the same amount of data. The results show that the proposed algorithm with a fairness index close to 1 outperforms all the other algorithms.

In Figure 5, we illustrate the impact of the number of transmit antennas on the performance of the different algorithms for the non-symmetric distributed users case. According to the results, the sum data rate remains almost constant except when using the max-min worst user algorithm. However, even with 32 users and 8 transmit antennas, the proposed algorithm is more suitable than the max-min worst user in practice.

\section{CONCLUSION}

In this paper, we have evaluated the impact of multiple transmit antennas on the performance of multicast OFDM systems by considering the proposed algorithm. We have shown that the resource allocation which includes the precoding vector selection, the subcarrier allocation and the bit loading is a difficult optimization problem. We have proposed a suboptimal algorithm that is able to extract almost all gain of multiple transmit antennas for the resource allocation by avoiding the optimization. This algorithm has been developed and performed at a subcarrier level. However, it can be also applied on clusters of subcarriers in order to reduce the feedback load.

Compared to multicast OFDM systems, we have shown that it is possible to increase the sum data rate with multiple transmit antennas by using suboptimal solutions. When the users are symmetrically distributed around the base station, the gain is rather small (15\%); on the other hand, when the users are non-symmetrically distributed, we have shown that the proposed algorithm outperforms OFDM systems with the subcarrier allocation. We have also obtained better performance with the proposed algorithm compared to the maxmin worst user for both symmetrically and non-symmetrically user distribution in the case of two transmit antennas without performing optimization. However, for more than 4 transmit antennas, the achievable gain of the max-min worst user is higher than the proposed algorithm in the expense of high complexity.

It is of interest to extend these results by evaluating the impact of the feedback and quantization errors on the performance of the proposed algorithm.

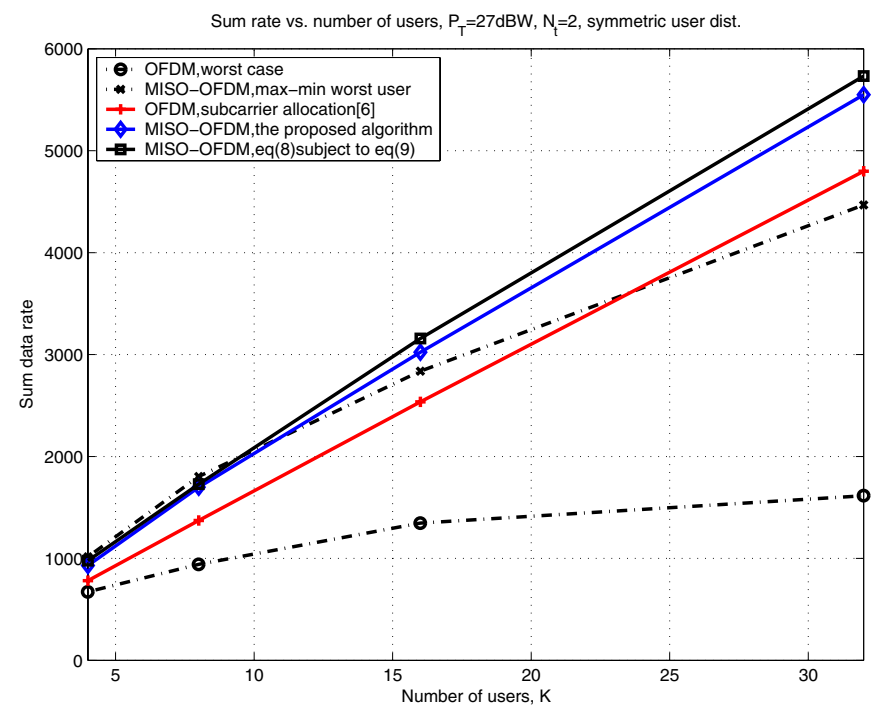

Fig. 3. Sum data rate versus the number of users for $P_{T}=27 \mathrm{dBW}$ and $N_{t}=2$ for symmetric user distribution.

\section{REFERENCES}

[1] U. Varshney "Multicast over Wireless Networks". Communications of the ACM, vol.45, pp. 31-37, Dec.2002.

[2] L. Cimini. "Analysis and simulation of a digital mobile channel using orthogonal frequency multiplexing". IEEE Trans. on Communication, vol. 33,pp. 665-675, 1985.

[3] G. J. Foschini, M. J. Gans. "On the limits of wireless communications in fading environment when using multiple antennas". Wireless Personal Communication, vol. 6,pp. 311-335, 1998.

[4] C. Y. Wong, R. S. Cheng, K. Ben Letaief, R. D. Murch. "Multiuser OFDM with adaptive subcarrier, bit and power allocation". IEEE Journal on Sel. areas in Comm., vol. JSAC-17,pp. 1747-1758, Oct. 1999.

[5] Z. Shen, J.G. Andrews, B. L. Evans. "Optimal Power Allocation in Multiuser OFDM Systems". IEEE Globecom, pp. 337-341, Dec. 2003. 


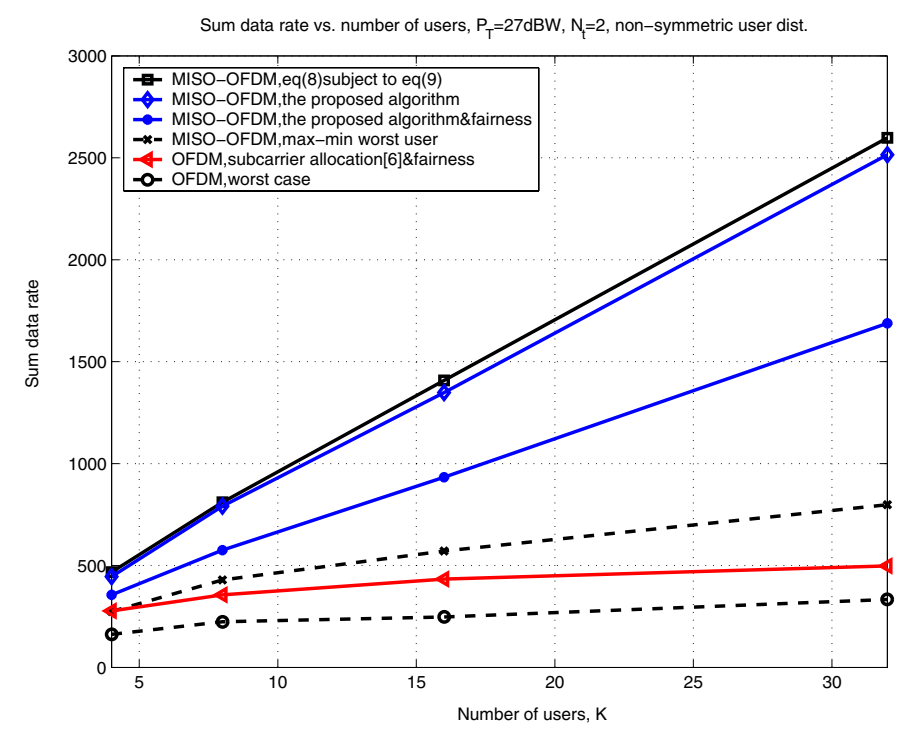

Fig. 4. Sum data rate versus the number of users for $P_{T}=27 \mathrm{dBW}$ and $N_{t}=2$ for non-symmetric user distribution.

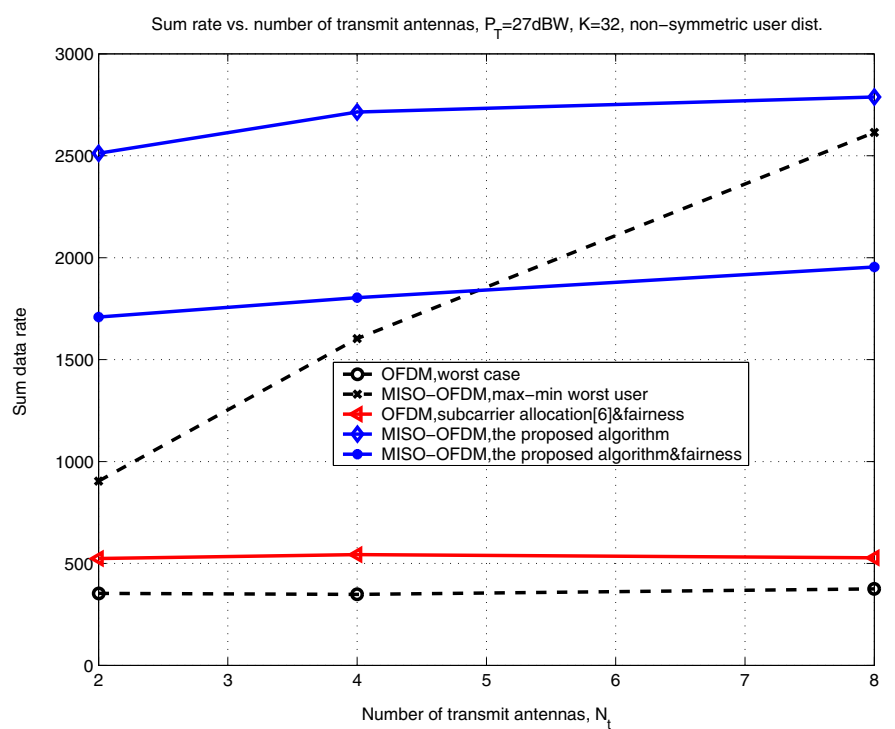

Fig. 5. Sum data rate versus the number of transmit antennas for $P_{T}=$ $27 \mathrm{dBW}$ and $K=32$ for non-symmetric user distribution.

[6] C. Suh, C. S. Hwang. "Dynamic subchannel and bit allocation for multicast OFDM Systems". Proc. of IEEE PIMRC'04, Sept 2004, Barcelone, Spain.

[7] J.W. Byers, M. Luby, M. Mitzenmacher. "A Digital Fountain Approach to Asynchronous Reliable Multicast". IEEE Journal on Sel. areas in Comm., vol. JSAC-20, pp. 1528-1540, Oct. 2002.

[8] J. G. Proakis "Digital Communication 3rd edition". New York:McGrawHill, 1995.

[9] N. D. Sidiropoulos, T. N. Davidson, Z. Q. Luo, "Transmit beamforming for physical layer multicasting", To appear in the IEEE Trans. on Signal Proc.

[10] R. Jain, D.M. Chiu, W.R. Hawe, "A Quantitative Measure of Fairness and Discrimination for Resource Allocation Shared Computer Systems", Digital Equipment Corporation technical report TR-301, 1984.

[11] J. Khun-Jush, P. Schramm, U. Wachsmann, F. Wenger, "Structure and Performance of the Hiperlan/2 Physical Layer", Proc. of the IEEE
VTC'99, June 1999, vol. 5, pp. 2667-2671, Amsterdam.

[12] ETSI Normalization Committee, "Channel Models for Hiperlan/2 in Different Indoor Scenarios", France, 1998. 